\title{
The Family Transformation Due to the Assisted Reproductive Technologies
}

\author{
Hemion Braho PhD
}

European University of Tirana

hemion.braho@uet.edu.al

\section{Doi:10.5901/ajis.2015.v4n2p235}

Abstract

This article aims to make a brief study in the field of bioethics particularly on the influence of reproductive medicine on the family. This change is not only represented as a "crisis" of the traditional model based on marriage between men and women and its durability, but also on the proposition of the new models that contribute to this crisis. This study brings confront between these models trying also to draw conclusions about possible future scenarios and the feared crisis of identity of future generations. The aim aof this article is to demonstrate that family is still a human resourse.

Keywords: Assisted Reproductive Technology, Motherhood surrogacy, Family structure, Modernity and tradition

\section{Introduzione}

Lo studio della bioetica che si concentra sulle tecniche di procrezione medicalmente assistita e sul loro sviluppo ha apportato cambiamenti, non solo nelle questioni connesse ai "modi di nascere", ma anche nelle relazioni sociali e in particolare il modo di concepire il nucleo familiare. Questo perchè si facilita la creazione di nuove forme familiari monoparentali, omoparentali e multiparentali, mettendo in ombra la cosiddetta famiglia tradizionale.

L'obiettivo di questo breve lavoro si concentra proprio sullo studio comparator e trasversali fra questi modelli di famiglia "vecchi" e "nuovi". L'intento è cercare di comprendere quali siano le reali dimensioni e le differenze fra tali modelli cercando di cogliere chiaramente ciò che di vero e falso si trasmette nei diversi dibattiti sull'argomento e di fissare le prospettive per il futuro. Tutto ciò si cercherá di raggiungerlo attraverso un metodo analitico-sociale d'analisi della struttura familiare con l'aiuto dello studio storico-comparativo.

\section{La Moltiplicazione delle Figure Genitoriali}

Le novità apportate dalle tecniche di fecondazione assistita pongono molti problemi: l'erosione della famiglia intesa non come modello storicamente determinato, ma come struttura umana fondamentale. È chiaro che la riproduzione umana, oltre agli aspetti biologici, dà origine a vincoli di parentela dal valore antropologico costituendo l'asse portante della familiarità, che permette all'uomo di crescere e sviluppare rapporti umani dai primi momenti della propria vita. La novità, anche sotto il profilo antropologico, resa possibile dalle nuove tecnologie riproduttive è la costruzione di un uomo solo, in grado di autodeterminarsi; tali tecniche tendono ad attivare un'antropologia dal "carattere irrelato della soggettività" (D’Agostino, 2003, 251).

Tutto ciò è in contrasto con l'essenza della natura umana e questa nuova visione dell'uomo si contraddice e nasconde delle falsità sia sul piano delle dinamiche riproduttive, poiché la riproduzione assistita richiede in ogni caso la figura del donatore di materiale genetico e del medico che esegue l'intervento, sia sul piano delle dinamiche sociali, dato che nessun individuo della società è in grado di autodeterminarsi totalmente, nonostante l'individualismo moderno eserciti un grande fascino a livello psicologico ed ideologico (Sgreccia, 2007, 26; Donati, 1999, 85). Saranno analizzate queste dinamiche al fine di comprendere nello specifico, come agiscano le nuove tecnologie nell'ambito familiare.

\section{La Prospettiva della Famiglia}

La famiglia è una struttura composta da una serie di relazioni articolate che si sviluppano all'interno di altre relazioni complesse; questi rapporti costruiti internamente ed esternamente ad essa, hanno cambiato significativamente i ruoli riconosciuti ad ogni componente, creandone altri diversi, o elimanondoli del tutto; ad esempio, si è cambiata la vision della donna sia sotto un profilo sociale sia, addirittura, sotto un profilo antropologico, complicando, così, la visione del 
mercato stesso, allargando nettamente i confini di ciò che oggi possiamo definire come famiglia; sostanzialmente, è cambiato il modo di essere e di concepire la struttura familiare. Si è di fronte al rischio della perdita dei valori di cui la famiglia è portatrice, arrivando a pensare che sia necessario "congelare" la struttura della famiglia tradizionale. II rischio è quello della perdita dell'importanza sociale della struttura familiare, con grosse difficoltá a trovare delle alternative; i ritmi e le logiche dalla società moderna impongono ai suoi consociati una rinuncia alla struttura familiare per poter realizzare una vita normale, non schizofrenica. L'epoca moderna ha dovuto considerare continuamente un ripensamento della relazione familiare, con lo sforzo del mantenimento della struttura tradizionale, anche nelle attuali mutazioni delle situazioni sociali, politiche ed economiche.

Analizzando la filosofia relativemente moderna, notiamo in Hegel un esempio che includeva nel sistema statale il fulcro della relazione familiare, perchè considerate come elemento basilare nella costruzione dello Stato, ed ancorandolo saldamente ad esso, con il risultato per la famiglia di trasformarsi totalmente fino a perdere ogni fondamento, con l'entrata in crisi il modello statale a cui si riferiva (Cavallera, 2006, 294: Cavallari, 1996, 101). Bisogna constatare, quindi, come la famiglia si sia ridotta oggi ad un recipiente vuoto che ospita al suo interno le forme relazionali e affettive basate su un fragile sentimento, legate in qualche modo alla sessualità e alla riproduzione, e dalla quale ci aspetta un certo livello 0 qualche forma di educazione o di solidarietà. Alla cosiddetta "famiglia tradizionale" o "nucleare", fondata sulla complementarietà sessuale e costruita sul matrimonio, che è evidentemente incentrata sull'educazione equilibrata dei figli e di conseguenza legata al future per la successione delle generazioni, oggi non gli viene più riconosciuta nessuna differenza sostanziale in relazione ad altri legami interpersonali.

A completamento di questa realtà interpersonale, vi è appunto lo sviluppo ormai inarrestabile delle tecniche di procreazione medicalmente assistita e di quelle di ingegneria genetica le quali rendono ancora necessario e attuale l'istituzione di un luogo generativo basato sulla complementarietà biologica. Infatti, non è più necessario ricorrere in senso fisico alla sessualità umana per poter avere dei figli e con la conseguenza di mettere da parte tutta quella serie di legami connessi a questa "necessità".

Secondo alcuni questo porta al vantaggio sociale di poter considerare forme nuove di relazioni, in un certo senso più immediate e spontanee e di certo più "leggere", indipendentemente dalle "costrizioni" di genere sessuale, dell'età e perchè no, anche della specie; di fatto siamo di fronte a relazioni nelle quali prevalgono il volere dell'uomo, eliminando ogni limite connesse alle imperfezioni e contingenze del biologico, che con i suoi limiti dovuti alla natura non contribuisce più in maniera decisiva nella definizione dell'umano. Le argomentazioni che vengono apportate a queste teorie si prospettano sia a livello teorico e filosofico, sia sul piano empirico: da una parte si sostiene che non esiste nessuna società naturale, includendo in questa affermazione anche la famiglia e si cerca, d'altra parte, di dimostrare come la famiglia sia da considerare come luogo di sottomissione e di annullamento del soggetto, costruendo questo pre-giudizio attraverso strategie pilotate. L'insieme di queste teorie, da un lato filosofiche e dall'altro di carattere empirico coincidono con la riproposizione della tesi per la quale, la libertà e l'autonomia della persona vengono lese quando si cerca di definire come natura ciò che appartiene semplicemente alla cultura tradizionale. Tutto ciò sembra essere in contrasto con il generale sentimento etico dei singoli consociati e che è assolutamente da combattere in una società volta alla promozione delle differenze e dell'autonomia dei singoli nelle loro scelte (Aramini, 1999, 121; Bornstein, 2002, 6).

Queste constatazioni nascondono in esse tutta una serie di errori, sia di carattere logico che di carattere filosofico, perchè confondono i concetti e i valori in gioco. L'autonomia, di cui si parla e il rispetto delle diversità, o la cosiddetta promozione dei singoli, portano ad una serie d'incongruenze, in una serie di livelli, compreso quello giuridico, presenti nei diversi codici, alle carte costituzionali, legati alla questione della famiglia.

Di quali errori stiamo parlando? La prima tesi ha una forte dose di ambiguità perchè non esiste nessuna società che sia di fatto puramente naturale, ma ciò non legittima la società ad essere contro natura, se si intende con questo termine un'entità sociale che esista completamente distaccata dai cambiamenti di carattere storico-sociale; pochi filosofi nel corso della storia hanno pensato alla famiglia in questo modo. Analizzando la seconda tesi, il fatto poi che in alcune società tale istituzione comportasse la cosiddetta inferiorità femminile, non significa che in date società tutto l'insieme dei legami, relazioni, o rapporti affettivi che vanno sotto la copertura della definizione di famiglia debbano essere considerate di fatto sbagliate: si può fare l'esempio della schiavitù che è una concezione molto rudimentale legata ad alcune società e alle loro relazioni economico-sociali, in tutte le epoche, ma questo non significa che società molto creative 0 sviluppate come quella dell'antica Roma 0 anche più recenti vadano considerate come negative 0 insignificanti. Insomma, la realtà familiare contiene sempre elementi legati alla natura, sviluppatasi nei confine dello spazio e del tempo dell'ambito che definiamo cultura. Sotto questo profilo, abbiamo creazioni naturali e istituzioni non-naturali, ma nel caso dell'uomo sono considerate tutte culturali. Di fatto il rapporto natura-cultura non può essere considerato solamente come antitetico, perchè esso è in grado di supportare i cambiamenti culturali degli elementi in relazione fra loro, senza dover 
forzatamente cadere nel relativismo culturale che, portando tutte comunicazioni interpersonali familiari ad equivalersi, le annulla; d'altro canto non si deve cadere nella concezione opposta basata sulla cultura forzata per la quale si congela la strutura della famiglia tradizionale con la conseguenza di concepire le relative relazioni con pessimiso e senso di resa al non-cambaimento.

Negli spazi delineati, si aprono alter strade, diverse dal concetto relativistico e da quello anacronistico. Una in particolare è legata a quella che di fatto molte famiglie vivono positivamente anche in una società complessa che fa parte del terzo millennio: è innegabile che molte famiglie funzionino almeno e più del suo contrario patologico. Non possiamo considerare solamente i dati sociologici come risolutivi; la famiglia difatti è si un dato di fatto sociale, uniti agli elementi già visti della sua natura, ma tutti questi dati si annullano di fronte alla tesi per cui la famiglia è da considerare l'insieme di relazioni affettive, sessuali ed educative di ogni suo componente, che di fatto esistono con tutti gli elementi esternamente considerati come positive o negativi.

Questa tesi dmostra che la struttura familiare viene confermata nel tempo dal suo "percorso storico" come un insieme che "deve esistere" perché possiamo vivere una vita equilibrate in relazione all'altro, sotto molteplici profile e in particolare quello affettivo-sessuale da un lato e quello parentale-affiliativo dall'altro. In questo senso, tutte le statistiche negative proposte, molte volte anche contraddittorie, potrebbero esser lette in maniera non distruttiva, se analizzate dal punto di vista proposto in precendeza.

Al giorno d'oggi è sicuramente più complicato e sociologicamente più difficile instaurare legami stabili e duraturi, anche per nostre colpe. Si potrebbe affermare che in una società individualistica diventa molto complicato affermare ed affermarsi in relazioni durature, ma anche perché, in postivo, è latente in tutti la consapevolezza e la necessità di un serio esercizio quotidiano di ciò che ci configura fondamentalmente come uomini, ovvero la libertà e la responsabilità. La combinazione qui descritta fra le categorie della libertà e della responsabilità, che dentro la famiglia fiorisce e si realizza pienamente nelle giuste relazioni tra i suoi membri e le alter forme società che possono essere di tipo politiche, professionali, amicali, economiche, sportive, volontariato, di appartenenza religiosa, etc. Tutto ciò è fondamentale al fine di garantire un'autonomia effettiva (che per l'uomo è sempre anche dipendenza riconosciuta, come ad esempio nel caso del bambino che obbedisce ai genitori), di fronte a forme di prevaricazione 0 sfruttamento; esattemente grazie al funzionamento della famiglia, riusciamo a riconoscere e a combattere le spinte nichilistiche. In questa prospettiva, vision con la quale si può osservare la famiglia può essere molteplice, e allo stesso modo devono essere sottolineati gli elementi positivi dati spesso per scontati o anche dimenticati, di fronte alla preoccupazione di casi che non rappresentanti di certo la normalità di questa relazione, e meno ancora la sua "normalità".

La famiglia rappresenta per tutti una risorsa, ed è infondato, di fatto, il pregiudizio che essa non possa più avere niente da dire e da dare alle persone e alle società al giorno d'oggi. La famiglia ed anche i ruoli in essa formatosi sono fondamentali per una adeguata formazione e un'armonica identità personale. Questo non soltanto nelle primi fasi della vita 0 solamente nella direzione che si muove dai genitori ai figli; la famiglia rimane indispensabile perchè fonda la stabilità e l'organicità del legame coniugale per uno sviluppo equilibrato dei componenti legati all'affettività, ritagliandosi un ruolo central nella preparazione e l'acquisizione di corretti elementi solidali e relazionali, che non sono strumentali, ma hanno un valore realmente significativo da considerare anche fuori dai confini della stessa famiglia; essa è il luogo dove si forma il giudizio delle persone inteso come sguardo critico alla realtà e ciò avviene tramite l'educazione che per essere completa ha bisogno alla base di legami seri e duraturi fra le persone che la compongono. Ciò che invece apportano le tecniche di fecondazione assistita o l'ingegneria genetica è l'opposto, perché oltre a destabilizzare l'equilibrio dei rapporti, rendendo ad esempio onnipotenti i genitori o uno di loro tramite la scelta di avere il figlio come e quando dicono loro, scompongono alla base la struttura familiare con l'intervento di terzi soggetti determinando la moltiplicazione delle figure genitoriali.

\section{Surrogazione di Maternità e Surrogazione di Paternità}

Nell'analisi degli effetti concreti delle tecniche di riproduzione artificiale si terrà maggiormente conto dell'angolo visuale riferito al bambino, che più di tutti subisce gli effetti di tali tecniche e ha meno potere di incidere su ciò che avviene.

Riassumendo le problematiche legate alla moltiplicazione delle figure genitoriali derivanti da tali tecniche, si ricorda che la surrogazione di paternità riguarda la tecnica della fecondazione eterologa in cui vi è una scissione fra padre genetico (il donatore di spermatozoi) e il padre sociale (colui che effettivamente si dovrà occupare del bambino); la surrogazione di maternità invece può portare addirittura a tre figure materne dove la scissione può esserci fra madre genetica (colei che dona il materiale biologico), madre surrogata (colei che porta avanti la gravidanza) e madre sociale (colei che si dovrà occupare del bambino). Ricordando le fattispecie di procreazione medicalmente assistita della donna 
se ne possono ricordare tre:

1) Il prelievo di ovociti: si ha a che fare con una gravidanza portata avanti da una donna responsabile dell'intera operazione, che ricorre al materiale genetico altrui per avere un figlio (scissione fra madre genetica e madre sociale);

2) La surrogazione di maternità: in questo caso il marito, previo ottenimento del consenso della compagna, procede alla fecondazione artificiale di una donna esterna alla coppia; quando il figlio viene partorito, viene consegnato alla coppia che ha dato vita alla pratica. In questo caso sia il materiale genetico che lae gestazione è a carico di una terza donna;

3) L'affitto d'utero: l'embrione questa volta è formato in laboratorio con il materiale genetico della coppia ed è impiantato poi in una terza donna; come nel caso precedente quando avviene il parto, il bambino viene consegnato alla coppia, quindi il materiale genetico è della donna, ma la gestazione è di una terza.

Di fronte a questo scenario in cui vi è una molteplicità di figure genitoriali di riferimento, potrebbe esserci un grave danno alla formazione dell'identità del bambino, con ripercussioni sia sul piano sociale sia psicologico, nonché su quello giuridico dove si verifica la liceità e la giustizia di tali pratiche. Proprio in ambito giuridico vi sono stati dei tentativi di risposta e di legittimazione a tali pratiche, sostenendo che la surrogazione di paternità e soprattutto la surrogazione di maternità, che presenta maggiori problemi pratici150, possono essere inquadrati nello schema del contratto a favore di terzo, vale a dire il bambino che verrà ad esistere. Questa soluzione lascia della perplessità sia perché si fa difficoltà ad inquadrare la nascita sotto uno schema contrattuale, sia perché tale soluzione elude il problema principale riguardante la pluralità di figure genitoriali per il bambino (Shalev, 1989, 103; Sgreccia, 2007, 509).

Una risposta a tali problematiche si tenta di fornirla tenendo segreta l'identità dei donatori (anche se nel caso della madre uterina è praticamente impossibile); l'anonimato di questi soggetti in molti paesi è garantito per legge e dovrebbe tutelare proprio il bambino. Un atteggiamento del genere serve sicuramente ad evitare dei problemi al bambino, anche se non risolve tali questioni, dato che la realtà in questo modo viene semplicemente offuscata e non viene apportata nessuna soluzione; infatti, tale fatto rimane presente almeno nella coscienza dei genitori e come conseguenza si riflette anche sul bambino con il quale i genitori sono costantemente in contatto. Facendo degli esempi concreti, è frequente il caso di coppie, che dopo avere praticato la fecondazione eterologa, entrino successivamente in crisi; in questo caso, nonostante la previsione di leggi che impediscono il disconoscimento di paternità, non si potrà impedire un disconoscimento del figlio in ambito privato e familiare, con conseguenze comunque gravi sul bambino e sulla famiglia nel complesso. La fecondazione eterologa, quindi, è piena di rischi sia per il figlio, sia per i genitori o aspirant tali.

Gli Stati Uniti hanno una lunga storia di approvazione delle tecniche di fecondazione eterologa, sono decisamente moltiplicati i casi di disconoscimento di paternità dopo aver ottenuto un figlio grazie all'eterologa. Molti padri "non naturali", che credevano di essere preparati ai figli che pensavano fossero a loro apparteneti, ma senza affinità genetica hanno trovato il nuovo nato molto "diverso" da loro considerandolo un estraneo, anche per il rapport esclusivo che si crea fra il figlio e la mamma "naturale" non sentendosi parte di questa relazione. Molti padri biologici hanno in realtà impressioni simili i primi tempi dopo un nuovo nato, a causa del legame stretto ed esclusivo, che si crea fra madre e bambino, ma è una difficoltà che si supera col tempo, anche se nei padri provvenienti dall'eterologa è più alta la percentuale di chi si considera in maniera permanente escluso.

Generalmente, il concepimento tramite fecondazione eterologa espone la coppia ad una resistenza più flebile di fronte alle difficoltà che immancabilmente si presentano lungo la strada per una famiglia, coinvolgendo spesso in maniera diretta 0 indiretta questioni connesse alla relazione genitori-figli, che hanno a che fare con l'educazione e la loro maturazione. In questo senso, "nella famiglia, gli scambi gestuali, verbali, emozionali, sono così intensi e ravvicinati che basta una pausa inattesa, un omissione un lapsus, un tono inconsueto per suscitare il sospetto e la paura.

\section{Conclusioni}

L'inquietante, il perturbante - insegna Freud - consiste nell'inserzione dell'estraneità nel cuore stesso della familiarità. Soltanto se lo sfondo familiare rimane sufficientemente rassicurante per il figlio può staccarsene senza provare eccessiva angoscia" (Vegetti Finzi, 1994, 238). Inoltre, con l'espansione dei test genetici e delle diagnosi prenatali, si renderà necessario se non doveroso il venir meno dell'anonimato dei soggetti che hanno donato il materiale genetico; tutto questo nel nome di una medicina che si contraddice e diventa sempre più confusa nel perseguire una salute che non riesce a far fronte alla pressione dei desideri (D'Agostino, 2003, 251; Sgreccia, 2007, 660; Cotta, 2004, 25; Mathieu, 2000, 117; Pavone, 2006, 1179; Neri, Serra, 2007, 463). 


\section{References}

Aramini M. (1999), La procreazione assistita: scoprire il senso di un nuovo modo di nascere, Roma, Paoline.

Baldini G., Cassano G., (2003), Persona, biotecnologie e procreazione, Cesano Boscone (MI), IPSOA.

Bornstein M. H., (2002), Handbook of Parenting: Being and Becoming a Parent, , New York (USA), Lawrence Erlbaum Associates.

Cotta S. (2004), Il diritto come sistema di valori, Cinisiello Balsamo (Milano), San Paolo.

D'agostino F., (1998), Bioetica, Torino, Giappichelli.

D'agostino, F. (2003), Una filosofia della famiglia, Milano, Giuffrè.

Mathieu, V. (2000)., L'uomo animale ermeneutico, Torino, Giappichelli.

Neri, G., SERRA, A., (1 dicembre 2007) La diagnosi prenatale oggi, in "La civiltà cattolica".

Pavone V. (Novembre-Dicembre 2006), II diritto ad essere sani. Un nuovo approccio alle biotecnologie mediche e riproduttive, in "II Mulino",

Rodotà, S. (a cura di) (1994), Questioni di bioetica, , Bari-Roma, Laterza.

Scabini, E., ROSSI, G., (1999) Famiglia 'generativa' o famiglia 'riproduttiva'? II dilemma etico nelle tecnologie di fecondazione assistita, , Milano, Vita e Pensiero.

Sciancalepore G., Stanzione P. (2001), Filiazione e procreazione assistita, Cesano Boscone (MI), IPSOA.

Sgreccia, E. (2007), Manuale di bioetica. Vol 1. Fondamenti ed etica biomedica, Milano, Vita e Pensiero.

Shalev, C. (1989), Birth power. The case of surrogancy, London, New Heaven.

Zatti P., (1989), 'Natura' e 'cultura' nella procreazione artificiale, in FERRANDO G., La procreazione artificiale tra etica e diritto, Padova, Cedam. 
